\section{Cahiers de Narratologie}

Analyse et théorie narratives

$28 \mid 2015$

Le récit comme acte cognitif

\title{
Une approche cognitive de la narrativité musicale
}

\section{Christian Hauer}

\section{(2) OpenEdition}

\section{Journals}

Electronic version

URL: https://journals.openedition.org/narratologie/7194

DOI: 10.4000/narratologie.7194

ISSN: 1765-307X

\section{Publisher}

LIRCES

Electronic reference

Christian Hauer, "Une approche cognitive de la narrativité musicale", Cahiers de Narratologie [Online], 28 | 2015, Online since 29 October 2015, connection on 21 September 2021. URL: http://

journals.openedition.org/narratologie/7194 ; DOI: https://doi.org/10.4000/narratologie.7194

This text was automatically generated on 21 September 2021.

Article L.111-1 du Code de la propriété intellectuelle. 


\title{
Une approche cognitive de la narrativité musicale
}

\author{
Christian Hauer
}

L'approche proposée par le titre de cet article est problématique aux deux sens du terme: la musique comme narrativité, un point parfois encore considéré comme problématique, et la musique comme cognition, soit la problématique même de l'article. Toutefois, en traitant celle-ci, il sera répondu à celui-là : traitée sous l'angle cognitif, la musique est nécessairement narrative. Pour plusieurs raisons, dont l'une est rarement évoquée, alors qu'elle constitue l'une des spécificités de la musique : celle-ci est exécutée par un narrateur, que nous appellerons ainsi car il raconte l'histoire de l'œeuvre. Et, point décisif, en situation de concert mais aussi d'enregistrement dans un studio, le narrateur raconte cette histoire à quelqu'un: un auditeur qui est là pour écouter l'histoire de l'œuvre qui va lui être racontée, et qui ne peut lui être racontée qu'en étant jouée. Cette histoire n'est pas seulement celle du discours musical lui-même, dans son aspect artisanal, elle est en même temps bien plus que cela : "expériencialité d'une conscience » - au sens de Fludernik (1996). Ou "expérience de vie réelle» à travers une conscience: une expérience, selon Fludernik, d'essence nécessairement narrative. Comment l'auditeur prend-il connaissance - au sens de cognitif - de cette expérience narrative de l'exécutant? Par quels mécanismes mentaux et corporels? Pour le dire comme Fludernik : en fonction de ses propres expériences. Afin de relier l'expériencialité de l'auditeur à celle du narrateurexécutant, nous introduirons le concept d'embodied simulation, ou d'empathie. Nous traiterons ainsi successivement de l'expériencialité comme facteur premier de la narrativité (notamment) musicale; du narrateur en musique comme lieu du sens, présence concrète, vivante, incontournable; enfin, de l'embodied simulation comme phénomène cognitif fondateur de l'expérience musicale - narrative - de l'auditeur.

\section{La narrativité comme « expériencialité »}

2 Le concept d'« expériencialité » de Fludernik (1996, Caracciolo 2013) est l'un des concepts fondateurs de la narratologie cognitive. Car il traite des modalités de 
l'actualisation d'un texte par un lecteur et, plus généralement, de « la relation cognitive entre l'expérience humaine et les représentations humaines de l'expérience " (Caracciolo 2013: § 1). En effet, pour Fludernik, la narrativité correspond à l'expériencialité définie comme «l'évocation quasi-mimétique de l'expérience de la vie réelle » $(1996: 12)$. C'est une narrativité de « nature anthropomorphique » : un texte est narratif non pas parce qu'il relate une intrigue, une condition non nécessaire, mais parce qu'il se réfère à « l'expérience de la vie réelle », qui s'inscrit dans un espace et un temps donnés (26). Point décisif, c'est « l'implication émotionnelle avec une telle expérience et son évaluation [qui] fournissent des points d'ancrage cognitifs pour la constitution de la narrativité » (13). Toute expérience, remémorée ou présente, est liée à une émotion (29).

3 Toutefois, deux orientations sont possibles: l'expériencialité comme propriété intrinsèque à la narrativité, donc «identifiable dans les textes ", ou comme processus cognitif propre à «l'interaction entre le texte et le lecteur» (Caracciolo 2013: § 2). Nous adopterons l'une et l'autre. D'abord, du point de vue de l'œuvre. Qui, quelle qu'elle soit, est imprégnée d'expériencialité. En amont même de toute lecture, de toute interprétation. En tant qu'inscription anthropologique - d'une époque, mais aussi comme cuvre d'un individu, comme manifestation d'une intention créatrice - qui peut être soumise à divers questionnements, sémiotiques, herméneutiques ou autres. Ensuite, du point de vue cognitif de l'actualisation de cette "expérience de la vie réelle»: expériencialité comme expérience d'une conscience qui est comprise par le lecteur en fonction de ses propres expériences, donc d'un cadre d'interprétation préexistant qui est celui de la vie réelle (Fludernik 1996:12). On reconnaît ce que vit l'autre personnage de fiction ou non - en fonction de ce que l'on vit et ressent soi-même.

Soulignons dès maintenant la fonction nodale de l'exécutant considéré comme narrateur: il propose d'une œuvre une expérience, une parmi beaucoup d'autres possibles, certes, mais qui est - concrètement et à ce moment précis - cette œuvre-là pour l'auditeur qui lui-même l'actualise en fonction de sa propre expérience au sens d'expériencialité. Et ce que représente le narrateur en musique ne peut faire sens pour l'auditeur qu'en projetant l'expérience qui lui est proposée ainsi dans un monde fictionnel. L'expérience au sens où l'entend Fludernik n'est pas une imitation, au sens de mimésis, mais une projection artificielle d'une structure sémiotique que le lecteur - ou l'auditeur - actualise au sein d'une réalité fictionnelle (Fludernik 1996 : 35 ; Caracciolo 2013 : § 11). Il ne peut le faire que par l'imagination. Surtout en musique, selon Walton, en raison du lien intime qui se noue entre écouter de la musique et imaginer faire l'expérience d'émotions, par exemple, plutôt que de percevoir quelqu'un les exprimer : «les expériences musicales ne sont pas simplement des expériences causées par la musique ; elles sont des expériences de musique » (1994 : 55).

\section{Le narrateur en musique}

\section{Narrateur vs. œuvre}

5 Le narrateur - l'exécutant - raconte l'histoire qu'est l'œuvre, lui donnant forme et expression, prenant en charge son actualisation. La partition apparait ainsi comme un ensemble d'instructions que l'exécutant doit déchiffrer, littéralement mettre en cuvre, au même titre qu'un acteur de théâtre pour le personnage qu'il incarne. Ou : l'exécutant est 
à l'histoire qu'est l'ouvre ce que, par exemple, le récit de fiction est à ce qu'il raconte. Ou encore : c'est le narrateur qui effectue l'opération essentielle de la configuration, qui fait passer le pré-texte au texte, la substance à la forme. C'est une spécificité de la musique : elle ne peut exister concrètement qu'exécutée (cf. Brétéché 2012: 54-59), par ce qui constitue un narrateur qui produit un discours de l'intérieur de l'œuvre. D'où le caractère incarné de la musique : des personnes en chair et en os - le ou les exécutants - racontent quelque chose à d'autres personnes en chair et en os - les auditeurs. Par conséquent, toute musique est indissociable de la figure de l'exécutant.

6 L'exécutant en musique remplit toutes les fonctions habituellement attribuées au narrateur. Selon le Handbook of Narratology, le narrateur, «c'est quelqu'un qui raconte à quelqu'un d'autre que quelque chose est arrivé » (Margolin 2014). Comme l'exécutant en musique, ce «quelque chose » qui « est arrivé » étant l'œuvre. Il est dit aussi que le narrateur « peut être envisagé comme un agent fictionnel qui fait partie du monde de l'histoire et dont la tâche est de rendre compte de l'intérieur d'événements qui dans ce monde sont réels ou effectifs pour lui » (\$9). L'exécutant-narrateur en musique fait "partie du monde de l'histoire " pour deux raisons au moins : c'est par lui que cette histoire qu'est l'œuvre prend forme, une forme, en fonction de son point de vue particulier à lui (ce qui est d'ailleurs un autre attribut du narrateur), et c'est à ce moment précis que l'exécutant devient interprète ; cette histoire a été écrite pour lui, pour qu'il la joue, pour qu'il la fasse sienne. Enfin, il rend bien compte « de l'intérieur d'événements qui dans ce monde sont réels ou effectifs pour lui ». Où l'on retrouve l'expériencialité, car il s'agit bien de cela: d'événements "réels ou effectifs pour lui»-pour lui le narrateur en musique.

7 Le narrateur en musique raconte l'œuvre en lui donnant la forme de sa propre expériencialité, en accord plausible avec celle qui fonde l'œuvre. Il donne existence à cette œuvre, en la donnant à écouter. Certes, ce narrateur est d'abord un récepteur qui met en sons sa proposition de l'œuvre - une proposition qui peut faire l'objet d'une approche herméneutique, en l'occurrence de la réception, en tant que réponse à des questions liées à un se-comprendre particulier devant l'œuvre (cf. Hauer 2007, 2009). Toutefois, par sa réception même, l'exécutant est d'abord un narrateur, car c'est lui qui donne forme à l'œuvre, en produisant un discours de l'intérieur de l'œuvre : il en est la condition nécessaire, car en musique, sans exécutant ou narrateur, il n'y a pas d'œuvre écoutable.

\section{Narrateur vs. performance}

8 L'exécutant en musique est donc un narrateur qui donne forme à l'œuvre par une performance, passée ou présente, enregistrée ou en direct. Une partition n'a pas d'existence en soi, sinon écrite. Certes, elle peut être entendue intérieurement en la lisant. Mais à la seule condition d'avoir déjà entendu de la musique. Quiconque n'a jamais entendu un instrument de musique utilisé pour la première fois est incapable de l'entendre intérieurement sur la seule base de la partition. Si le théâtre qui, du point de vue narratologique, partage un grand nombre de traits communs avec la musique, peut être lu ou entendu au moyen du même langage (le théâtre est d'ailleurs bien plus souvent lu qu'entendu, c'est-à-dire joué), il en va tout autrement avec la musique, qui ne peut être lue qu'avec des compétences spécifiques qui n'ont rien à voir avec le fait d'écouter la musique. Même pour Boulez qui, tout en soulignant l'importance de 
«l'écoute intérieure » pour composer, «afin de pouvoir imaginer le résultat sonore sans aucun intermédiaire instrumental », «[1]a musique doit être entendue » (Boulez et al. 2014 : 173, 169). Lire la musique ne permet pas de tout entendre, en raison de la nature indocile des timbres instrumentaux. Un compositeur, encore : «Une partition peut se lire (devrait pouvoir se lire) en musique. Pour Boucourechliev, ce n'est vrai qu'avant le premier baroque, avant que les instruments n'interviennent de façon décisive dans le rendu de l'œuvre, avant que le timbre ne soit devenu ce que j'appelle un "vecteur de composition" " (Courtot 2014).

9 La performance est ainsi une condition nécessaire pour qu'il y ait musique : l'exécutantnarrateur utilise les instructions fournies par la partition (lorsqu'elle existe), voire par d'autres sources (comme les traités théoriques pour les musiques anciennes), pour être au plus près des intentions de l'œuvre. Ce caractère nécessairement performé de la musique est central, car il explique aussi, nous y reviendrons, pourquoi l'exécutantnarrateur en musique est le lieu du sens pour toute musique entendue (cf. Hauer 2014). Ce qui est médiatisé ainsi par ce narrateur à travers la performance, donc par sa présence concrète, visuelle et auditive ou seulement auditive, c'est une expérience de l'ouvre qui est expression d'une conscience. En même temps, la performance d'une œuvre écrite est la performance par un narrateur d'une histoire - celle de l'œuvre - qui est passée, à la fois au sens de trace d'une expérience passée - celle du compositeur - et de clos, terminé, inchangeable. Ce temps au passé est le temps narratif par excellence, qui agit comme un signal de la narrativité (cf. Wolf 2014 : 134). En effet, « les histoires, y compris leur fin, sont arrivées [...] avant d'être racontées, performées ou représentées " et elles " suscitent le "sens de la préséance des événements" ou du caractère passé de l'histoire en question» (129).

10 En résumé : la performance est, en musique, ce qui institue l'exécutant comme narrateur. D'une part, par rapport à l'œuvre, puisque c'est par sa performance que le narrateur donne une forme qui correspond à son expérience de cette œuvre : le narrateur raconte en fonction de son présent à lui une œuvre qui se présente au passé. D'autre part, par rapport à l'auditeur: la performance est la médiation qui rend possible une relation cognitive entre l'auditeur et l'expérience de l'œuvre proposée par le narrateur. Notamment par le phénomène de l'embodied simulation. Mais avant d'aborder cette question, il faut répondre à celle-ci : quelles sont les conditions qui rendent une telle relation cognitive possible? Ou: quel est le cadre général dans lequel une telle relation peut prendre forme?

\section{Performance et « concept transmédial de la fictionnalité »}

Considérée sous l'angle à la fois narratif et cognitif, la performance en musique relève de ce que Zipfel (2014) appelle le "concept transmédial de la fictionnalité ». Qui décrit moins ce qu'est la fictionnalité que ce qui la rend possible quel que soit le type de média considéré. Zipfel en distingue trois couches superposées.

D'abord, le «monde fictionnel ». Un monde ne peut être dit fictionnel que s'il présente des différences - ou un « certain degré de déviation »- avec le monde réel (105-6). C'est le principe même du phénomène de l'expériencialité tel que nous l'avons défini en musique: comme expérience projetée dans une structure sémiotique - l'œuvre ou l'improvisation telle que performée par le narrateur - qui ne peut être actualisée que par l'imagination au sein d'une réalité fictionnelle. 

Zipfel en appelle à Walton, pour qui le faire-semblant est l'attitude qu'un public adopte à l'égard des œuvres fictionnelles : à l'instar du jeu, notamment les jeux d'enfants, il y a engagement complet et conscience que ce monde - du jeu ou de l'œuvre d'art - n'est pas le monde réel (106-7). La relation entre exécutant et auditeur est de cet ordre: du faire semblant que ce qui se passe est vrai. Ainsi, les émotions ressenties sont vraies, sans être réelles, étant artificielles, sans lien direct avec la vie quotidienne.

Enfin, parmi les règles connues et acceptées de ce jeu du faire-semblant: la «pratique institutionnelle». L'attitude fictionnelle et de faire-semblant adoptée par le public est déclenchée par le fait qu'il reconnaît chez le "producteur " l'intention de lui faire adopter une telle attitude: cette "entente mutuelle", fondée sur des règles et conventions connues et acceptées, est la condition nécessaire pour toute relation de type cognitif (108). Les « bénéfices » d'une telle relation constituent le but recherché par le public : pourquoi accepterait-il sinon de consacrer une partie de son temps, de son énergie et de ses émotions à se "tracasser " avec de la fiction, plutôt que de les réserver à des applications plus pratiques (109) ? La fiction musicale s'inscrit dans ces deux cas de figure: sans cette "entente mutuelle», même a minima, la performance musicale ne peut aboutir à une quelconque relation cognitive et donc à aucune expériencialité. Telle que celle de l'embodied simulation, qui pose la question du couplage entre perception et action.

\section{L'embodied simulation comme condition d'une cognition narrative}

\section{Un détour par les arts visuels}

Ce couplage entre perception et action chez l'observateur a fait l'objet de nombreuses études en neurosciences. Freedberg (historien de l'art) et Gallese (neuroscientifique) ont posé l'hypothèse d'une relation, chez l'observateur, entre les " embodied empathetic feelings » et les traces laissées par les gestes de l'artiste : ils s'appuient sur l'exemple de deux tableaux d'art abstrait (de Pollock et de Fontana), où les gestes de l'artiste, qui sont seulement implicites à travers les traces laissées sur les toiles, sont ressentis par le spectateur dans son propre corps (2007). Cette hypothèse, fondée sur le principe des neurones miroirs, sera par la suite expérimentée de manière plus précise. Umiltà et son équipe (2012) s'appuient sur la comparaison entre des œuvres de Fontana et une version simplifiée de ces œuvres : le cortex moteur est activé, mais uniquement à la vue des œuvres dans leur version première. D'où cette conclusion : il s'agit là d'une forme d'embodied simulation, puisque la vue des lacérations sur les toiles active les représentations motrices liées au même geste dans le cerveau de l'observateur. Avec des œuvres de Kline, Sbriscia-Fioretti et son équipe (2013) parviennent à une conclusion analogue: la vue des coups de pinceau suscite chez l'observateur l'activation des zones sensori-motrices qui contrôlent les actes moteurs menant à la production de ces coups de pinceau. Autrement dit, leur perception n'est possible que si l'on sait comment faire pour atteindre ce résultat. Les auteurs soulignent ainsi la fonction de l'embodied simulation des gestes de l'artiste dans la perception des œuvres d'art.

Ces études s'intéressent à des tableaux non figuratifs, ce qui, du point de vue de la narrativité en musique, n'est pas anodin. Dans les deux cas, il manque une histoire, une 
référence, un lien concret avec le réel, c'est pourquoi on qualifie souvent d'art abstrait aussi bien les tableaux non figuratifs que la musique instrumentale. Toutefois, l'embodied simulation n'a rien d'abstrait: elle est une condition préalable à toute expérience, donc à toute narrativité, dépassant ainsi le seul cadre de la chose racontée en tant que telle, même dans un texte de fiction. Car c'est par l'embodied simulation qu'elle peut prendre forme - qu'elle accède à l'expériencialité. Et, parmi les facteurs contribuant à l'embodied simulation, si ce n'est le principal, il y a le «mécanisme miroir ».

\section{Le mécanisme miroir}

17 Le système des "neurones miroirs" a été découvert dans les années 1990, par Rizzolatti et son équipe (cf. Rizzolatti et Sinigaglia [2006] 2011, Rizzolatti et Destro 2008, Mukamel et al. 2010, Oztop et al. 2012). En voici le principe: lorsque nous regardons quelqu'un exécuter un acte, les neurones miroirs, qui se trouvent dans le cortex moteur préfrontal, déchargent comme si nous exécutions l'acte nous-mêmes. Autrement dit, notre système moteur - l'action - et nos fonctions sensorielles - la perception - s'instruisent mutuellement. En 2010, Rizzolatti a déclaré « qu'il serait plus exact de parler des neurones miroirs en termes de mécanisme de base du système nerveux présent dans différentes parties du cerveau qui, tout en maintenant une connexion directe entre information sensorielle et activation motrice, change de fonction spécifique selon l'aire dans laquelle elle se trouve"; d'où le titre de sa conférence: «Le mécanisme miroir: un mécanisme neuronal pour comprendre les autres » (Sofia $2011: 238-9$ ).

Il s'agit donc d'un système ou d'un mécanisme qui permet de " comprendre les autres ». Du moins leurs intentions (cf. Iacoboni et al. 2005, Fogassi et al. 2005, Juan et al. 2013, Kohler et al. 2002). C'est une implication importante du mécanisme miroir : permettre « de corréler les mouvements observés à nos propres mouvements et d'en reconnaître la signification ", notre cerveau étant ainsi capable de comprendre immédiatement les actions accomplies par les autres « sans avoir recours à aucun type de raisonnement, en se fondant uniquement sur ses propres compétences motrices » (Rizzolatti et Sinigaglia [2006] $2011: 10$ ). C'est en fonction de notre propre répertoire d'actes moteurs que nous comprenons ce que nous voyons et entendons, que nous comprenons les actions et les intentions d'autrui.

De nombreuses études expérimentales s'appuient sur cette hypothèse pour souligner l'interaction entre perception et action, ou compréhension du monde extérieur et de ce qui s'y passe par nos capacités de mimétisme - de simulation incarnée, motrice. La vue de coups de brosse peints éveille les représentations de l'action correspondante chez l'observateur (cf. Taylor et al. 2012, Leder et al. 2012, Ticini et al. 2014). Ou celle de photographies montrant des personnages en action (cf. Proverbio et al. 2009). La littérature n'est pas en reste (cf. Wojciehowski et Gallese 2011, Gallese 2010, Keen 2006 et 2013, Herman 2011, Speer et al. 2009), ni le cinéma (cf. Gallese et Guerra 2012) ou encore la musique, depuis l'étude fondatrice de Molnar-Szakacs et Overy (2006). Citons l'étude de Peckel et al. (2014), qui montre qu'une musique perçue en fond sonore par un auditeur « résonne avec le système moteur » de celui-ci et influence, en fonction des capacités motrices des membres utilisés (marcher, taper avec les doigts, etc.), les mouvements qu'on lui demande pourtant d'effectuer sans tenir compte de la musique qu'il entend. Nous évoquerons avec Hyman (2012) trois études marquantes: lorsque des danseurs 
observent des mouvements relevant de la danse dont ils sont spécialistes, les aires cérébrales associées à la préparation et à l'exécution de ces mouvements sont nettement plus actives que dans tout autre cas; à l'écoute d'une pièce qu'ils savent jouer, les pianistes activent les aires cérébrales associées à la perception auditive et - ce qui n'est pas le cas pour une pièce familière mais qu'ils ne savent pas jouer - les aires cérébrales associées à la préparation de l'action ; regarder une partition active chez les musiciens - contrairement aux non-musiciens - les aires du cerveau associées à la planification motrice.

\section{Transition}

Traiter du système des neurones miroirs - ou du mécanisme miroir - amène ainsi à traiter de l'embodied simulation. Toutefois, l'inverse n'est pas nécessairement vrai : nous ne dirons pas que le phénomène de l'embodied simulation s'explique par le seul mécanisme miroir. Nous soulignerons surtout le lien très fort - dont le mécanisme miroir est un acteur important - entre perception et action, dans la mesure où toute perception est action. Plus précisément: où toute perception implique qu'elle déclenche dans le même temps des processus moteurs.

21 Il s'agit de la première phase de l'embodied simulation (qui correspond aux "inverse models»). Et si l'embodied simulation permet de faire accéder ce qui est perçu au rang d'expérience, elle permet aussi de préparer ce qui suit, de se projeter vers (ce qui correspond aux «forward models»). C'est ce que nous appellerons le «nœud narratif » de l'embodied simulation, abordé ici du point de vue de la musique.

Mais revenons d'abord sur le principe de l'embodied simulation, afin d'en proposer une définition plus précise, sur deux plans au moins: l'implication de l'émotion dans l'embodied simulation, sans laquelle ce qui se passe ne serait pas expérience au sens où nous l'entendons ici - comme expérience de la vie réelle; le moment du déclenchement même de l'embodied simulation - moment de tension, d'une "tension pathétique " (Sevilla 2014).

\section{L'embodied simulation : définition}

"Simulation incorporée »: voilà la traduction littérale. Qui rend bien compte de ce phénomène: quelque chose qu'on incorpore à soi en le simulant, au sens d'imiter. Ce qui correspond aussi, chez de nombreux auteurs, à ce qui est appelé empathie, contagion émotionnelle ou mimétisme. Ces termes renvoient au même type de phénomène, même si le terme d'embodied simulation est plus précis sur un point essentiel : l'incorporation de ce qui est perçu. Dans tous les cas, la musique est « ancrée dans le corps» (Imberty 2013) et la question qui se pose est de déterminer, selon le titre d'une étude de Gallese et Sinigaglia : « How the Body in Action Shapes the Self» (2011).

Proposons la définition suivante de l'embodied simulation: « un mécanisme fonctionnel qui fait que les actions, les émotions ou les sensations que nous observons activent nos propres représentations internes des états du corps qui sont associés à ces stimuli sociaux, comme si nous étions engagés dans une action similaire ou comme si nous expérimentions une émotion ou une sensation semblable » (Freedberg et Gallese 2007 : 198). Autrement dit, nous comprenons ce que nous voyons ou ce que nous entendons en le simulant à partir de ce que nous avons déjà expérimenté. Ce qui intègre également l'émotion, 
y compris en musique. Nous renverrons à l'article de Molnar-Szakacs et Overy (2006), au titre évocateur, que l'on traduira ainsi: "Musique et neurones miroirs: du mouvement à l'émotion » (cf. aussi Becker 2010), ou à des études portant sur le rapport entre l'émotion provoquée par la musique et l'activation des neurones miroirs (cf. Koelsch 2005, Koelsch et al. 2006, 2010). L'émotion est un facteur important, sinon essentiel, du faire-semblant qui correspond à l'engagement complet qu'un auditeur, par exemple, manifeste à l'égard d'une cuvre fictionnelle telle que proposée par un narrateur-exécutant dans une relation cognitive d'« entente mutuelle». Pour le dire comme Walton, l'empathie consiste à imaginer ressentir ce qu'autrui - personnage réel ou de fiction - semble ressentir, ce que nous appliquons en musique à la figure - même physiquement absente - de l'interprète, contrairement à Walton, pour qui la musique pousse à imaginer faire l'expérience de certaines émotions, mais le plus souvent à partir d'elle-même (1994: 58-9). Selon Juslin, la « contagion émotionnelle » est l'un des huit mécanismes psychologiques à la base de l'émotion en musique, qui consiste pour un auditeur à percevoir l'émotion exprimée par la musique et à la mimer intérieurement (cf. Juslin 2013, Juslin et al. 2013, 2010).

Pour désigner un phénomène analogue (qu'il n'applique pas à la musique), Sevilla parle de «tension pathétique », définie « comme l'immersion cognitive de l'émetteur et/ou le récepteur dans le pathos (émotions et sensations) d'un autre, symbolique ou réel, en adoptant sa perspective» (2014: § 14). Plus loin, l'auteur centre son propos sur "l'immersion cognitive de l'empathie : modalité plus intense, mais pas exclusive, de la tension pathétique » (ibid.). Et d'ajouter qu'une « certaine idée de tension » participe de cette empathie, dans la mesure où celle-ci suppose une « réaction obligée au stimulus de départ » (\$15). Selon Baron-Cohen, « il y a au moins deux étapes dans l'empathie : reconnaissance et réponse » (ibid.). C'est un point important, car l'empathie, ou embodied simulation, ne se déclenche pas automatiquement: la réponse et son intensité dépendent des attentes et des capacités cognitives du récepteur (de même que de ses "aptitudes éthiques », cf. § 22-3). Pour reprendre, en le traduisant, le titre d'une remarquable étude : l'empathie et la contagion émotionnelle constituent un lien entre les émotions reconnues et les émotions ressenties à l'écoute de la musique (Egermann et McAdams 2013). L'empathie constitue un indicateur de préférence: elle sera d'autant plus intense que l'auditeur apprécie la musique qu'il entend, étant amené «à être plus attentif aux émotions exprimées et à ressentir du coup une empathie accrue » (151). D'autres facteurs interviennent également, comme le genre, l'attention portée à ce qui est écouté et le degré d'expertise musicale.

Nous sommes désormais en mesure d'examiner les deux phases croisées de l'embodied simulation: de la perception (réelle) à l'action (simulée) - «inverse model»- et de l'action (réelle) à la perception (simulée) - « forward model ».

\section{Musique et embodied simulation : de la perception (réelle) à l'action (simulée)}

Nous nous appuierons sur une étude de Cox (2011), qui présente une hypothèse sur la manière dont la musique est intériorisée dans les corps et les esprits des auditeurs. C'est « l'hypothèse mimétique », qui « aborde la question de l'embodiment en montrant comment l'imagerie musicale - souvenir, anticipation ou encore penser à de la musique - relève en partie d'une imagerie motrice » qui est "liée aux efforts et aux 
mouvements produits par notre système squeletto-moteur », ce qui « implique aussi les efforts suscités par la performance musicale » (\$2). Nous comprenons en partie la musique en fonction d'un mimétisme, "d'une empathie physique qui consiste à imaginer que nous produisons les sons que nous entendons » (\$3).

Une approche analogue est développée par Pignocchi (2012) : «l'expérience de toute œuvre d'art prend nécessairement forme autour d'une reconstruction mentale de la démarche de l'artiste », essentiellement «à notre insu» (18). Ainsi, « nous percevons certaines propriétés de l'œuvre comme si nous les avions nous-mêmes produites " (ibid.). D'où l'hypothèse d'une " "simulation consciente" de la démarche de l'artiste ", qui concerne « les propriétés de l'œuvre que l'observateur perçoit, grâce à son propre savoir-faire, directement comme le résultat d'intentions qu'il aurait pu lui-même former» (175). Savoir se servir d'un crayon à papier "suffit à doter l'observateur d'associations visuo-motrices lui permettant de percevoir immédiatement les traits d'un dessin comme le résultat d'actions » (170).

Comme Pignocchi, Cox propose de nombreux exemples, sur la base de dix-huit " principes » associés à cette hypothèse :

\section{Préliminaire : les sons et leurs sources}

1. Les sons sont produits par des événements physiques : les sons indiquent (signifient) leur source physique.

2. Beaucoup ou la plupart des sons musicaux sont la preuve des actions motrices humaines qui les produisent.

\section{Le comportement mimétique et l'imagerie en général}

3. Les êtres humains comprennent d'autres entités (animées ou non, humaines ou non) et les événements au sein d'un environnement en partie par imitation explicite et implicite.

4. L'imitation explicite et implicite constitue des représentations corporelles des actions observées.

5. Les êtres humains comprennent le comportement d'autrui en partie par le comportement mimétique et la MMI (" mimetic motor imagery»).

6. Les actions imaginées sont informées par les actions effectuées.

7. L'imitation peut être intentionnelle, consciente et souvent non consciente.

8. L'imitation est plus fortement activée dans l'observation des actions dirigées vers un but.

Les principes plus spécifiques à la musique

9. L'action mimétique et la MMI se produisent en temps réel et en « rappel » (et peutêtre dans la «planification»).

10. L'imagerie motrice mimétique et l'action se produisent selon trois modalités : intramodale, intermodale, et amodale.

11. Toutes les caractéristiques acoustiques peuvent être ou seront représentées par mimétisme.

12. Différents types de musique «invitent» à différents types d'engagement mimétique.

13. Certaines musiques atténuent l'invitation mimétique.

14. La musique d'ensemble offre plusieurs « invitations ».

15. Les réponses mimétiques sont souvent plus fortes dans des contextes live que dans des contextes enregistrés.

16. La MMI varie en force et en précision selon les individus. 
17. La MMI motive et limite la conceptualisation (métaphorique ou autre).

18. Le comportement mimétique et la MMI impliquent la participation mimétique, la communication, et l'affect ».

31 C'est sans doute en musique que ce phénomène mimétique, ou embodied simulation, est le plus frappant. Parce que la musique est nécessairement jouée et qu'il y a donc toujours quelqu'un qui fait - un exécutant. La musique ne peut exister concrètement qu'exécutée, même si la notion d'exécution tend à s'atténuer dans le cas, par exemple, des musiques générées en réseau sur internet. Mais, dans tous les cas, la musique, c'est du son, c'est-àdire un artefact produit intentionnellement par quelqu'un et un ensemble de vibrations ressenties de manière empathique et liées à une source sonore plus ou moins identifiable. La figure de l'exécutant - au sens le plus large - est ainsi présente physiquement dans toute musique entendue, y compris enregistrée.

C'est pour cette raison que la musique est diegesis - racontée - et mimesis - montrée : racontée, car il y a un narrateur qui raconte non ce que dit l'œuvre, mais l'œuvre ellemême ; montrée, car incarnée, mimée par le narrateur-exécutant. La musique suscite donc en permanence l'expérience de l'embodied simulation. Entre les musiciens et les auditeurs. Et entre les musiciens, car quel plus bel exemple d'embodied simulation - ou d'empathie - en acte que des musiciens qui jouent, et plus encore improvisent ensemble?

Toutefois, cette approche doit être complétée par une autre : comment la manière dont nous incorporons les sons nous permet de préparer et de rendre plus efficace notre expérience de la musique. Si l'embodied simulation permet de vivre la musique que nous entendons (et faisons), elle permet aussi de se projeter vers ce qui suit. Elle apparaît ainsi comme un nœud narratif articulant le maintenant et l'après, donnant sens à l'un pour le projeter vers l'autre.

\section{Musique et embodied simulation : de l'action (réelle) à la perception (simulée)}

Nous nous appuierons sur une étude de Maes et son équipe (2014), qui distingue deux approches de l'embodied simulation en musique. D'une part, les « inverse models », qui correspondent au flux d'information menant de la perception à l'action, permettant d'associer aux informations entrantes les stimulations motrices correspondantes (1). D'autre part, les «forward models ", qui correspondent au flux d'information menant de l'action à la perception, donnant ainsi la possibilité de prévoir l'issue sensorielle probable d'une action planifiée ou exécutée (2). Ils préparent les processus perceptuels et permettent donc de donner forme à l'expérience musicale (4). Considérant que la combinaison de ces deux modèles permet de comprendre comment nous interagissons avec le monde extérieur, les auteurs proposent de les associer afin de comprendre comment le système moteur humain et ses actions influencent la perception musicale (2).

Cette étude distingue trois implications des «forward models »: l'« atténuation »l'exécution d'une action pour laquelle on peut prédire les conséquences sensorielles atténue la perception de ce qui est effectivement entendu (5-6); la «facilitation »- le mouvement lié à la musique, comme la danse, facilite la perception du temps (6). Et la «désambigüisation» (7-8): la planification et l'exécution de mouvements corporels permettent de prévoir les conséquences sensorielles des actions et ainsi d'affiner la 
perception et la compréhension de la musique, ce qui concerne aussi les émotions que celle-ci exprime (cf. aussi 9-10), en précisant que l'observation de mouvements corporels (voir des musiciens jouer) influence tout autant la perception de la musique produite que le fait de produire ces mouvements ou de les planifier.

À partir de là, un point reste à souligner : le concept de «modèles internes » (« internal models»). Il découle de "l'hypothèse associative », selon laquelle le mécanisme miroir n'est pas seulement le résultat de l'évolution humaine, mais aussi celui de "l'apprentissage sensori-moteur associatif »: "grâce à des expériences répétées de manière systématique, des événements sensoriels sont associés à des actes moteurs particuliers" et "des liens "excitateurs" sont créés entre les deux, entraînant le développement de modèles internes " (3). Cette co-activation opère automatiquement dans les deux cas énoncés plus haut: dans les sens perception-action et action-perception (ibid., cf. aussi 9).

Deux précisions : ces modèles internes ne peuvent se construire que grâce à la capacité du cerveau à évoluer, y compris physiologiquement, pour automatiser des tâches répétitives, comme pour la pratique musicale (cf., notamment, sur la plasticité cérébrale, Peretz et Zatorre 2005, Paquette et Mignault-Goulet 2014, Schön 2013); ils peuvent être assimilés à des scripts sensori-moteurs, donc à des scripts dynamiques - des embodied scripts. Ils sont source de narrativité, car à ces scripts sont associés des sensations, des ressentis, des émotions, des images, des gestes, des représentations, etc. - des mouvements indissociablement corporels et mentaux, ancrés dans l'expérience de la vie réelle. Où nous retrouvons l'expériencialité.

\section{Conclusion}

La musique est donc récit à plusieurs titres. D'abord, parce qu'il y a en musique un narrateur au sens narratologique du terme : l'exécutant, indispensable pour qu'il y ait musique - sans performance, pas de narrateur, ni musique possible. Cet exécutant est le lieu $d u$ sens en musique: il lui donne forme, existence, la rend présente à lui-même et aux autres. Il raconte non pas ce que dit l'œuvre, mais l'œuvre elle-même.

Et ce, pour lui permettre de raconter. Car la musique est récit - elle peut raconter. Non pas des histoires, certes, au sens d'un récit de fiction. Nous renverrons à deux grands pionniers de la narrativité musicale. D'abord, Tarasti: «c'est dans les termes d'une intrigue abstraite que l'on devrait considérer la signification qu'une œuvre tente de nous transmettre » : il ne s'agit pas de « démontrer que la musique est capable d'énoncer des récits spécifiques, mais expose plutôt en quoi des structures de la musique peuvent être associées à des récits » (2007: 209, cf. aussi 2006 [2002]). Ensuite, Grabócz : «nous appellerons narrativité musicale le mode d'organisation des signifiés à l'intérieur d'une forme musicale » (2007: 241, cf. aussi 1986, 2009, 2011). Enfin, il faut mentionner, outre les travaux de Hatten (1994, 2004), l'ouvrage majeur publié par Almén (2008).

Si la musique est récit, elle l'est aussi d'un point de vue cognitif. Ce qui n'est possible que par la performance. Elle est seule capable de créer les conditions de la fictionnalité, fondée sur l'adhésion - le faire-semblant - d'un auditeur à l'idée que l'œuvre musicale institue un monde fictionnel: condition nécessaire de toute relation cognitive. Qui comprend au moins deux plans. 
D'abord, l'actualisation de l'œuvre par l'auditeur - sa compréhension. Il peut être rendu compte de ce processus d'actualisation cognitive par le concept de «tension narrative » (Baroni 2007, 2010), qui s'applique à la musique (cf. Baroni 2011, Grabócz 2011). C'est la tension narrative qui rythme l'intrigue, en contrastant temps forts et temps faibles, tensions et résolutions. Elle fait en sorte que le lecteur (ou l'auditeur) est accroché, surpris, en un mot noué par l'intrigue. C'est ce que Sevilla, qui prolonge et précise les travaux de Baroni sur la tension narrative, appelle la «tension télique», qu'il applique à la musique et qu'il définit comme "l'immersion cognitive de l'émetteur et/ou du récepteur dans l'inconnue relative aux intentions d'un sujet, agent ou patient, vis-à-vis d'un fait qui n'a pas encore été accompli » (2014 : § 25).

Ensuite, c'est cette interaction forte entre l'auditeur et la performance qui ouvre la voie à l'embodied simulation et à ses différents aspects, des nœuds narratifs constitués par le croisement entre les « inverse » et les « forward models»- perception et action réelles vs. action et perception simulées - jusqu'aux embodied scripts. C'est là que l'expérience musicale peut devenir expériencialité, c'est-à-dire une "expérience de vie réelle » à travers une conscience - une expérience d'essence narrative (cf. Fludernik 1996).

C'est ainsi que la musique apparaît, à de multiples titres, comme un récit et comme un récit cognitif. Récit sans histoire, certes. Mais, à la suite d'Almén, nous considérerons comme une force, y compris sur le plan de la narrativité, ce que la musique ne peut pas faire: elle laisse à l'auditeur une plus grande liberté pour suivre les rapports narratifs entre les événements musicaux (cf. 2008: 13). Et surtout celle de s'attacher à l'essentiel : à ce qui fait œuvre, expérience, expériencialité.

\section{BIBLIOGRAPHY}

Almén, Byron (2008), A theory of musical narrative, Indiana University Press.

Baroni, Raphaël (2007), La Tension narrative. Suspense, curiosité et surprise, Paris, Éditions du Seuil.

- (2010), « Réticence de l'intrigue », in Narratologies contemporaines. Approches nouvelles pour la théorie et l'analyse du récit, Pier, John \& Berthelot, Francis (dir.), Paris, Éditions des Archives Contemporaines, p. 199-213.

- (2011), «Tensions et résolutions : musicalité de l'intrigue ou intrigue musicale ? », Cahiers de Narratologie [En ligne], 21 / 2011, mis en ligne le 21 décembre 2011. URL : http:// narratologie.revues.org/6461 (consulté le 13 avril 2012).

Becker, Judith (2010), « L'action-dans-le-monde. Émotion musicale, mouvement musical et neurones miroirs ", Cahiers d'ethnomusicologie, n² 23, p. 40-45. URL: http:// ethnomusicologie.revues.org/961 (consulté le 11 décembre 2012).

Boulez, Pierre \& Changeux, Jean-Pierre \& Manoury, Philippe (2014), Les neurones enchantés. Le cerveau et la musique, Paris, Odile Jacob. 
Brétéché, Sylvain (2012), « Des actes et des corps. Pour une corporéisation des actes musicaux », in Ontologies de la création en musique. Des actes en musique, Esclapez, Christine (dir.), Paris,

L'Harmattan, p. 45-72.

Caracciolo, Marco (2013), « Experientiality », in the living handbook of narratology, Hühn, Peter \& Pier, John \& Schmid, Wolf \& Schönert, Jorg (dir.), 7 novembre 2013, révisé le 24 décembre 2013, Hamburg, Hamburg University. URL: http://www.lhn.uni-hamburg.de/article/experientiality (consulté le 6 février 2014).

- (2014), « Those Insane Dream Sequences. Experientiality and Distorted Experience in Litérature and Video Games ", in Storyworlds accross Media. Toward a Media-Conscious Narratology, Ryan, MarieLaure \& Thon, Jan-Noël (dir.), Lincoln and London, University of Nebraska Press, p. 230-249.

Courtot, Francis (2014), courriel du 10 septembre 2014 à C. Hauer, 2 pages.

Cox, Arnie (2011), « Embodying Music: Principles of the Mimetic Hypothesis », Music Theory in Line, a journal of the Society for Music Theory, $n^{\circ} 17$ (2), p. 1-24. URL: http://www.mtosmt.org/ issues/mto.11.17.2/mto.11.17.2.cox.html - Beginning.

Egermann, Hauke \& McAdams, Stephen (2013), « Empathy and Emotional Contagion as a Link Between Recognized and Felt Emotions in Music Listening », Music Perception, Volume 31, Issue 2, p. 139-156.

Fludernik, Monika (1996), Towards a 'Natural' Narratology, London and New York, Routledge.

Fogassi, Leonardo \& Ferrari, Pier Francesco \& Gesierich, Benno \& Rozzi, Stefano \& Chersi, Fabian \& Rizzolatti, Giacomo (2005), « Parietal Lobe : From Action Organization to Intention Understanding ", Science, 308 (29 April 2005), p. 662-667.

Freedberg, David \& Gallese, Vittorio (2007), « Motion, emotion and empathy in esthetic experience », Cognitive Sciences, Vol. n 5, p. 198-203. Doi : 10.1016/j.tics.2007.02.003.

Gallese, Vittorio (2010), « The Mirror Neuron Mechanism and Literary Studies : An Interview with Vittorio Gallese », California Italian Studies, 2 (1).

Gallese, Vittorio \& Guerra, Michele (2012), « Embodying Movies : Embodied Simulation and Film Studies ", Cinema : Journal of Philosophy and the Moving Image, 3, p. 183-210.

Gallese, Vittorio \& Sinigaglia, Corrado (2011), « How the Body in Action Shapes the Self », Journal of Consciousness Studies, 18, n 7-8, p. 117-143.

Grabócz, Márta (1986), Morphologie des œuvres pour piano de Liszt. Influence du programme sur l'évolution des formes instrumentales, Paris, Kimé.

- (2007), « La narratologie générale et les trois modes d'existence de la narrativité en musique », in Sens et signification en musique, Grabócz, Márta (dir.), Paris, Hermann Éditeurs, p. 231-252.

- (2009), Musique, narrativité, signification, Paris, L'Harmattan.

- (2011), « Métamorphoses de l'intrigue musicale (XIXe-XXe siècles) », Cahiers de Narratologie [En ligne], 21 / 2011, mis en ligne le 21 décembre 2011, URL : http://narratologie.revues.org/6503 (consulté le 13 avril). Doi : 10.4000/narratologie.6503

Hatten, Robert (1994), Musical Meaning in Beethoven : Markedness, Correlation, and Interpretation, Bloomington and Indianapolis, Indiana University Press.

- (2004), Interpreting Musical Gestures, Topics, and Tropes : Mozart, Beethoven, Schubert, Bloomington and Indianapolis, Indiana University Press. 
Hauer, Christian (2007), « Pour une herméneutique de la création et de la réception musicales », in Sens et signification en musique, Grabócz, Márta (dir.), Paris, Hermann Éditeurs, p. 123-132.

- (2009), «L'herméneutique comme expérience universelle et science du langage musical. - De Schleiermacher à Hjelmslev, via Ricœur », in Semiosis et hermeneia : le sens langagier du musical, Vecchione, Bernard \& Hauer, Christian (dir.), Paris, L'Harmattan, p. 235-249.

- (2014), « Le narrateur en musique comme lieu du sens », in Ontologies de la création en musique. Des lieux en musique, Esclapez, Christine (dir.), Paris, L'Harmattan, p. 97-122.

Herman, David (2011), « Cognitive Narratology », in the living handbook of narratology, Hühn, Peter \& Pier, John \& Schmid, Wolf \& Schönert, Jorg (dir.), Hamburg, Hamburg University. URL : http:// www.lhn.uni-hamburg.de/article/cognitive-narratology-revised-version-uploaded-22september-2013 (consulté le 28 octobre 2012).

Hyman, Ira (2012), « Listening to Music and Watching Dance Using Mirror Neurons », Psychology Today / Mental Mishaps, p. 1-2.

Iacoboni, Marco \& Molnar Szakacs, Istvan \& Gallese, Vittorio \& Buccino, Giovanni \& Mazziotta, John C. \& Rizzolatti, Giacomo (2005), « Grasping the Intentions of Others with One's Own Mirror Neuron System », PLoS Biol, 3/3, e79, 0001-0007. Doi: 10.1371/journal.pbio.0030079.

Imberty, Michel (2013), « Mouvement, geste et figure : la musique ancrée dans le corps », in Expression et geste musical, Kogler, Susanne \& Olive, Jean-Paul (dir.), Paris, L'Harmattan, p. 25-36.

Juan, Elsa \& Frum, Chris \& Bianchi-Demicheli, Francesco \& Wang, Yi-Wen \& Lawis, James W. \& Cacioppo, Stephanie (2013), « Beyond human intentions and emotions », Frontiers in Human Neuroscience, Mars, Volume 7, Article 99, 14 pages. Doi : 10.3389/fnhum.2013.00099.

Juslin, Patrick N. (2013), « From everyday emotions to aesthetic emotions : Towards a unified theory of musical emotions ", Physics of Life Reviews, 10, p. 235-266.

Juslin, Patrick N. \& Liljeström, Simon \& Västfjäll, Daniel \& Lundqvist, Lars-Olov (2010), « How does music evoke emotions ? Exploring the underlying mechanisms », in Handbook of Music and Emotion. Theory, Research, Applications, Juslin, Patrick N. \& Sloboda, John A. (dir.), Oxford, Oxford University Press, p. 605-642.

Juslin, Patrick N. \& Harmat, László \& Eerola, Tuomas (2013), « What makes music emotionally significant, Exploring the underlying mecanisms ", Psychology of Music, 22 Août, publié en ligne avant impression. URL : http://pom.sagepub.com/content/early/2013/08/22/0305735613484548 (consulté le 2 juin 2014). Doi : 10.1177/0305735613484548.

Keen, Suzanne (2006), «A Theory of Narrative Empathy », Narrative, Volume 14, $n^{\circ} 3$, Octobre, p. 207-236.

- (2013), « Narrative Empathy », in the living handbook of narratology, Hühn, Peter \& Pier, John \& Schmid, Wolf \& Schönert, Jorg (dir.), Hambourg, Hamburg University. URL : http://www.lhn.unihamburg.de/article/narrative-empathy (consulté le 9 juillet 2013).

Koelsch, Stefan (2005), « Investigating Emotion with Music. Neuroscientific Approaches », Annals New York Academy of Sciences, $n^{\circ}$ 1060, p. 412-418. Doi: 10.1196/annals.1360.034.

Koelsch, Stefan \& Fritz, Thomas \& v. Cramon D., Yves \& Müller, Karsten \& D. Friederici, Angela (2006), « Investigating Emotion With Music : an fMRI Study », Human Brain Mapping, 27, p. 239-250. Doi : 10.1525/MP.2013.31.2.139. 
Koelsch, Stefan \& Siebel, Walter \& Thomas, Fritz (2010), « Functional Neuroimaging », in Handbook of Music and Emotion. Theory, Research, Applications, Juslin, Patrick N. \& Sloboda, John A. (dir.), Oxford, Oxford University Press, p. 314-344.

Kohler, Evelyne \& Keysers, Christian \& Umiltà, M. Alessandra \& Fogassi, Leonardi \& Gallese Vittorio \& Rizzolatti, Giacomo (2002), « Hearing Sounds, Understanding Actions : Action Representation in Mirror Neurons ", Science, 297, p. 846-848.

Leder, Helmut \& Bär, Siegrun \& Topolinski, Sascha (2012), « Covert Painting Simulations Influence Aesthetic Appreciation of Artworks », Psychological Science OnlineFirst, Vol. 23, $\mathrm{n}^{\circ}$ 12, p. 1479-1481. URL : http://www.ncbi.nlm.nih.gov/pubmed/23137968. Doi :

$10.1177 / 0956797612452866$.

Maes, Pieter-Jan \& Leman, Marc \& Palmer, Caroline \& Wanderley, Marcelo M. (2014), « Actionbased effects on music perception ", Frontiers in Psychology, Volume 4, Article 1008, Janvier, 12 pages. Doi: 10.3389/fpsyg.2013.01008.

Margolin, Uri (2012), « Narrator », in the living handbook of narratology, Hühn, Peter \& Pier, John \& Schmid, Wolf \& Schönert, Jorg, (dir.), Hamburg, Hamburg University. URL : hup.sub.unihamburg.de/lhn/index.php?title=Narrator\&oldid=2046, (consulté le 4 novembre 2012).

Molnar-Szakacs, Istvan \& Overy, Katie (2006), « Music and mirror neurons : from motion to 'e'motion », SCAN, I, p. 235-241. Doi :10.1093/scan/nsl029.

Mukamel, Roy \& Ekstrom, Arne D. \& Kaplan, Jonas \& Iacoboni, Marco \& Fried, Itzhak (2010), «Single-Neuron Responses in Humans during Execution and Observation of Actions », Current Biology, 20 (April 27), p. 750-756. Doi : 10.1016/j.cub.2010.02.045.

Oztop, Erhan \& Kawato, Mitsuo \& Arbib, Michael A. (2012), « Mirrors neurons : Functions, mechanisms and models », Neuroscience Letters, p. 1-13. Doi : 10.1016/j.neulet.2012.10.005.

Paquette, Sébastien \& Mignault Goulet, Geneviève (2014), « Lifetime benefits of musical training », Frontiers in Neuroscience, Avril, volume 8. Doi : 10.3389/fnins.2014.00089.

Peckel, Mathieu \& Pozzo, Thierry \& Bigand, Emmanuel (2014), « The impact of the perception of rhythmic music on self-paced oscillatory movements », Frontiers in Psychology, Volume 5, Article 1037. URL : http://journal.frontiersin.org/journal/10.3389/fpsyg.2014.01037/full. Doi : 10.3389/ fpsyg.2014.01037.

Peretz, Isabelle \& Zatorre, Robert J. (2005), « Brain Organization for Music Processing », Annual Review of Psychology, 56, p. 89-114.

Pignocchi, Alessandro (2012), L'œuvre d'art et ses intentions, préface de Jean-Marie Schaeffer, Paris, Odile Jacob.

Proverbio, Alice Mado \& Riva, Federica \& Zani, Alberto (2009), « Observation of Static Pictures of Dynamic Actions Enhances the Activity of Movement-Related Brain Areas », PLoS ONE, Volume 4, Issue 5, e5389. URL : http://www.ncbi.nlm.nih.gov/pubmed/19421311. Doi : 10.1371/ journal.pone.0005389.

Rizzolatti, Giacomo \& Sinigaglia, Corrado (2011), Les neurones miroirs [2006], traduit par Marilène Raiola, Paris, Odile Jacob Poches (1 ère édition : 2008).

Rizzolatti, Giacomo \& Fabbri Destro, Maddalena (2011), « Mirror neurons », Scholarpedia 3/1 (2008, dernière modification 21 octobre 2011), 2055. URL : http://www.scholarpedia.org/article/ Mirror_neurons (consulté le 9 janvier 2013). Doi : 10.4249/scholarpedia.2055. 
Sbriscia, Beatrice \& Berchio, Cristina \& Freedberg, David \& Gallese, Vittorio \& Umiltà, Alessandra (2013), « ERP Modulation during Observation of Abstract Paintings by Franz Kline », Plos One, Volume 8, Issue 10, 12 pages. Doi : 10.1371/journal.pone.0075241.

Schön, Daniele (2013), « Pratique musicale et plasticité cérébrale », in Le cerveau mélomane, Bigand, Emmanuel (dir.), Paris, Belin, p. 87-98.

Sevilla, Gabriel (2014), « La triple tension narrative : chrono-topique, pathétique, télique », Cahiers de Narratologie [En ligne], 26, mis en ligne le 17 septembre 2014. URL : http:// narratologie.revues.org/6901 (consulté le 20 octobre 2014). Doi : 10.4000/narratologie.6901.

Sofia, Gabriele (2011), « L'effet d'organicité de l'acteur : une hypothèse entre théâtre et neurosciences ", in Le surgissement créateur. Jeu, hasard ou inconscient, Journeau, Véronique Alexandre (dir.), préface de Menene Gras Balaguer, postface de Danièle Pistone, Paris, L'Harmattan, p. 233-253.

Speer, Nicole K. \& Reynolds, Jeremy R. \& Swallow, Khena M. \& Zacks, Jeffrey, M. (2009), « Reading Stories Activates Neural Representations of Visual and Motor Experiences ", Psychological Science, Volume 20, nº, p. 989-999.

Tarasti, Eero (2006), La musique et les signes. Précis de sémiotique musicale [2002], trad. fr., Paris, L'Harmattan.

- (2007), « La musique comme art narratif », in Sens et signification en musique, Grabócz, Márta (dir.), Paris, Hermann Éditeurs, p. 209-230.

Taylor, J. Eric \& Witt, Jessica K. (2014), « Listening to music primes space : pianists, but not novices, simulate heard actions ", Psychological Research, publié en ligne le 8 février 2014, 8 pages. Doi : 10.1007/s00426-014-0544-x.

Ticini, Luca F. \& Rachman, Laura \& Pelletier, Jérôme \& Dubal, Stéphanie (2014), « Enhancing aesthetic appreciation by priming canvases with actions that match the artist's painting style ", Frontiers in Human Neuroscience, Juin, Volume 8, Article 391. URL : http://www.ncbi.nlm.nih.gov/ pmc/articles/PMC4043134/. Doi : 10.3389/fnhum.2014.00391.

Umiltà, Alessandra \& Berchio, Cristina \& Sestito, Mariateresa \& Freedberg, David \& Gallese, Vittorio (2012), « Abstract art and cortical motor activation : an EEG study », Frontiers in Human Neuroscience, Novembre, Volume 6, Article 311, 9 pages. Doi : 10.3389/fnhum.2012.00311.

Walton, Kendall (1994), « Listening with Imagination : Is Music Representational ? », The Journal of Aesthetics and Art Criticism, Vol. 52, n 1, p. 47-61. URL : www.jstor.org/stable/431584 (consulté le 8 novembre 2014).

Wojciehowski, Hannah \& Gallese, Vittorio (2011), « How Stories Make Us Feel : Toward and Embodied Narratology », California Italian Studies, 2 (1).

Wolf, Werner (2014), « Framings of Narrative in Literature and the Pictorial Arts ", in Storyworlds accross Media. Toward a Media-Conscious Narratology, Ryan, Marie-Laure \& Thon, Jan-Noël (dir.), Lincoln and London, University of Nebraska Press, p. 126-147.

Zipfel, Frank (2014), « Fiction across Media : Toward a Transmedial Concept of Fictionality », in Storyworlds accross Media. Toward a Media-Conscious Narratology, Ryan, Marie-Laure \& Thon, JanNoël (dir.), Lincoln and London, University of Nebraska Press, p. 103-125. 


\section{ABSTRACTS}

This paper hypothesizes a cognitive approach of musical narrativity : from the cognitive angle, music is necessarily narrative. In this perspective, the paper discusses three main points. (1) Experienciality, in the meaning of "real life experience " through a consciousness, is, according to Fludernik, necessarily narrative. This concept is here applied to music. (2) In music, there is a narrator who tells the work, by a performance. He appears as the place of meaning. He is the concrete and essential presence so that there is music, or experienciality. (3) The embodied simulation (or empathy) appears like a cognitive phenomenon founding the musical and narrative experience of the listener, since it allows him to mimic physically the experienciality proposed by the narrator-performer. To address these points, this paper also approaches questions as essential as the mirror mechanism and thus reciprocal relationships between perception and action (see « inverse » and « forward models »), and the fictionality in music.

Cet article pose l'hypothèse d'une approche cognitive de la narrativité musicale, en ce sens que traitée sous l'angle cognitif, la musique est nécessairement narrative. Dans cette perspective, l'article traite de trois points principaux. (1) L'expériencialité, au sens d'une "expérience de vie réelle » à travers une conscience, est, selon Fludernik, nécessairement narrative, et ce concept est ici appliqué à la musique. (2) Il y a en musique un narrateur qui raconte l'œuvre, par la performance, et il apparaît ainsi comme le lieu du sens, présence concrète et indispensable pour qu'il y ait musique, ou expériencialité. (3) L'embodied simulation (ou empathie) apparait comme un phénomène cognitif fondateur de l'expérience musicale et narrative de l'auditeur, puisqu'il permet à celui-ci de simuler en l'incorporant l'expériencialité proposée par le narrateur-exécutant. Pour traiter de ces différents points, l'article abordera également des questions aussi essentielles que celles du mécanisme miroir, et donc des relations réciproques entre perception et action (cf. les «inverse » et « forward models »), et de la fictionnalité en musique.

\section{INDEX}

Mots-clés: narrateur, narrativité, cognition, performance, expériencialité, embodied simulation, empathie, mécanisme miroir, fictionnalité

\section{AUTHOR}

\section{CHRISTIAN HAUER}

Université de Lille

Centre d'Étude des Arts Contemporains (EA 3587)

Professeur en musicologie à l'Université Lille 3, dont il a été le premier vice-président de 2006 à 2012, et où il dirige le Centre d'Étude des Arts Contemporains (EA 3587) depuis 2012. Il a coédité avec Bernard Vecchione Le sens langagier du musical. « Semiosis » et " hermeneia » (L'Harmattan, 2009). Auteur d'une thèse de doctorat sur le Deuxième Quatuor op. 10 de Schoenberg, il est l'auteur de nombreux articles sur l'herméneutique de la création et de la réception musicales. Une édition de ces articles sous la forme d'un ouvrage est en préparation sous le titre Traité informel d'herméneutique musicale. Il prépare également, avec Christine Esclapez, une édition des articles publiés par Daniel Charles dans la revue Corps écrit entre 1992 et 1990. Il est membre de l'European Narratology Network depuis 2012 et oriente ses recherches sur la question de la narrativité dans les arts, et tout particulièrement la musique, en lien notamment avec les sciences cognitives. 